\title{
Case Report Form Annotation Guideline
}

National Cancer Institute

\section{Source}

National Cancer Institute. Case Report Form Annotation Guideline. NCI Thesaurus. Code C115749.

Documentation describing the process to map and assign subject information from a case report form to the proper positions within a dataset. 